\title{
Causas de reintervención quirúrgica en pacientes con fijación lumbar intervenidos por estenosis de canal
}

\author{
A. Gómez de la Riva; A. Isla; C. Pérez-López; R. Ortega; J.C. Fernández-Miranda y J. Heredero
}

Servicio de Neurocirugía. Hospital Universitario La Paz. Madrid.

Resumen

Objetivo. En el presente trabajo analizamos las causas por las que se ha debido reintervenir a pacientes diagnosticados de estenosis de canal que habían sido tratados mediante descompresión quirúrgica y fijación lumbar.

Material y métodos. Seleccionamos 74 pacientes entre enero de 1996 y diciembre de 2000 con un seguimiento mínimo de 4 años.

Resultados. $16(21,1 \%)$ fueron sometidos a una nueva intervención quirúrgica. La principal causa fue la estenosis de niveles adyacentes superiores a la instrumentación (en el 50\%). Otras causas fueron la fibrosis perirradicular, la persistencia de estenosis tras la cirugía, el dolor neuropático, la desconexión del sistema de fijación, la compresión radicular por tornillos transpediculares y la rotura de tornillos.

Conclusiones. Las complicaciones relacionadas con la técnica quirúrgica son bajas ya que la mitad de las reintervenciones en pacientes con estenosis del canal lumbar está producida por la estenosis del segmento superior a la fijación.

PALABRAS CLAVE: Estenosis lumbar. Instrumentación. Reintervención. Estenosis del nivel superior.

Causes of reoperations in patients with lumbar spinal stenosis treated with instrumentations

\section{Summary}

Objective. In present study we analyzes the causes leading to reoperation patients treated for lumbar spinal stenosis with laminectomy and lumbar instrumentation.

Material and methods. 74 patients operated between January of 1996 and December of 2000 with a minimum 4 year follow-up were seleted for the study.

Recibido: 19-08-05. Aceptado: 25-09-05
Results. 16 patients $(21$ 1\%) underwent a new surgical intervention. The main cause for reoperation was stenosis of the adjacent level superiorly to the instrumentation (in 50\%). Other causes were radicular fibrosis, persistence of stenosis after the surgery, neuropatic pain, failure of the instrumentation system, transpedicular screws misplacement and break of screws.

Conclusions. Complications related with the initial surgical procedure are low, as half of the reoperations were due to stenosis of the segment superior to the fixation.

KEY WORDS: Lumbar spinal stenosis. Instrumentation. Reoperation. Adjacent level.

Introducción

La estenosis del canal lumbar es un cuadro definido como cualquier tipo de estrechamiento del canal raquídeo, de los canales de las raíces nerviosas o de los agujeros intervertebrales ${ }^{3}$. Este cuadro tiene diversas etiologías como alteraciones congénitas como la acondroplasia, postraumática o metabólica, sin embargo, la causa más frecuente de estenosis de canal es la degenerativa; además, el envejecimiento progresivo de la población y el aumento de la esperanza media de vida hacen que se incremente paulatinamente la incidencia de los cuadros producidos por la degeneración de la columna lumbar.

Las técnicas quirúrgicas están basadas en la descompresión de los segmentos estenóticos, a lo que se puede añadir la fusión posterolateral para estabilizarlos dichos segmentos, con o sin instrumentación consistente en una fijación con tornillos transpediculares o fusión intersomática. Las teóricas ventajas de una fusión instrumentada son las mejores tasas de fusión con menor riesgo de pseudoartrosis, la disminución del tiempo de rehabilitación y una mejora clínica del paciente ${ }^{23}$.

En nuestro trabajo se trata el análisis de las causas de reintervención quirúrgica en los pacientes diagnosticados por estenosis de canal con o sin espondilolistesis 
degenerativa, en los que el tratamiento de entrada ha sido una descompresión con laminectomía más o menos amplia dependiendo de la estenosis y una fusión posterior instrumentada con tornillos transpediculares.

\section{Material y métodos}

De los 165 pacientes intervenidos por estenosis de canal, entre enero de 1996 y diciembre de 2000, en 74 casos el tratamiento considerado fue de fijación transpedicular, además de la descompresión quirúrgica de la zona estenótica. Se realizó un seguimiento retrospectivo mínimo de 4 años (rango de 48-60 meses).

En total se colocaron fijaciones lumbares en 30 hombres $(40,5 \%)$ y 44 mujeres $(59,5 \%)$, con una relación hombre/mujer de 1:1,5. La edad media de estos pacientes fue de 58,8 años (rango de 38 a 73).

La totalidad de los pacientes presentaban clínica y estudios de imagen compatible con estenosis de canal de predominio central. Además, 37 pacientes $(50 \%)$ presentaban espondilolistesis degenerativa de grado I-II. La clínica más frecuentemente presentada en los pacientes fue la claudicación neurógena en el $56,8 \%$ de los pacientes, seguido por la lumbociática bilateral en el $42,3 \%$ de los pacientes y la lumbociática de predominio unilateral en el $37,8 \%$ de los pacientes. También presentaron lumbalgia mecánica sin irradiación en el $12,2 \%$, parestesias o alteraciones sensitivas en el $27 \%$, déficit motor en el $13,5 \%$ e impotencia en el $1,4 \%$ de los pacientes.

La descompresión quirúrgica realizada fue una laminectomía completa con facetectomía bilateral a uno o varios niveles según la estenosis del paciente. La fijación lumbar consistió en una instrumentación con tornillos transpediculares de uno o más niveles con control radiológico para la adecuada colocación de los tornillos transpediculares. En todos los casos se colocó injerto óseo posterolateral autólogo de cresta iliaca o de apófisis espinosa y lámina del segmento estenótico. La posición del paciente en la mesa de operaciones fue sobre un trineo con flexión de las piernas entre $45^{\circ}$ y $60^{\circ}$.

Se colocó una instrumentación lumbar en un nivel en el $66,2 \%$ de los pacientes, siendo más frecuente en el nivel L4-L5, constituyendo el 48,6\% de todos los casos. Dos niveles se fijaron en el 30,5\% de los pacientes, siendo la instrumentación L3-L4-L5 la más frecuente con el 17,5\% de todas las fijaciones. En el 3,3\% se colocaron sistemas de 3 niveles (tabla 1).

\section{Resultados}

De estos 74 pacientes, 16 fueron sometidos a una nueva reintervención quirúrgica $(21,6 \%)$. Se reintervinieron 6 hombres y 10 mujeres con una relación hombre/mujer
Tabla 1

Relación de niveles intervenidos y reintervenidos posteriormente

\begin{tabular}{|l|c|c|c|r|}
\hline Nivel/niveles & $\mathbf{N}^{\circ}$ & $\mathbf{\%}$ & reintervenidos & \% \\
\hline L3-L4 & 6 & 8,1 & 1 & 6,3 \\
\hline L4-L5 & 36 & 48,6 & 7 & 43,7 \\
\hline L5-S1 & 7 & 9,5 & 3 & 18,7 \\
\hline L3-L5 & 13 & 17,6 & 1 & 6,3 \\
\hline L3-S1 & 1 & 1,3 & 1 & 6,3 \\
\hline L4-S1 & 8 & 10,8 & 2 & 12,5 \\
\hline L2-L5 & 2 & 2,7 & 1 & 6,3 \\
\hline L2-L4 & 1 & 1,3 & & 100 \\
\hline Total & 74 & 100 & 16 & \\
\hline
\end{tabular}

de 1:1,7. La edad media de los pacientes reintervenidos fue de 60 años (rango de 40 a 72). Las causas fueron: estenosis de canal en niveles superiores o inferiores a la instrumentación en 8 pacientes $(10,8 \%)$, fibrosis perirradicular en 1 paciente $(1,35 \%)$, persistencia de estenosis tras la cirugía en 1 paciente $(1,35 \%)$ dolor neuropático en 2 pacientes $(2,7 \%)$, desconexión del sistema de fijación en 1 paciente $(1,35 \%)$, compresión radicular por tornillos transpediculares en 2 pacientes $(2,7 \%)$ y rotura de tornillos en 1 paciente $(1,35 \%)$.

En total se retiraron 4 instrumentaciones $(5,4 \%)$, dos en pacientes por estenosis del nivel superior en las que se retiró la instrumentación en el mismo acto quirúrgico que la descompresión del nivel superior al comprobarse una artrodesis sólida. Las otras dos se retiraron en pacientes con dolor neuropático tras observarse una adecuada fusión y previo a la colocación de un neuroestimulador.

La mayor frecuencia de reintervenciones fue en el nivel L4-L5 con un 43,7\%, seguido por la fijación L5-S1 con un $18,7 \%$ de todas las reintervenciones. Los otros niveles tuvieron una menor frecuencia (tabla 1).

Dividimos las complicaciones en precoces y tardías.

\section{Complicaciones precoces}

\section{A. Malposición de tornillos transpediculares}

Dos pacientes fueron reintervenidos de forma precoz por malposición de tornillos. En el primero, el cuadro comenzó tras la cirugía presentando un déficit motor L5 derecho, tras fijación L5-S1 y laminectomía, con imposibilidad para la flexión dorsal y primer dedo del pie derecho sin afectación sensitiva. Se realizaron Rx y TAC en los que se observó un posible compromiso de la raíz en el receso lateral derecho por el tornillo 
transpedicular inferior derecho. Se reintervino al paciente retirando los tornillos del lado derecho $\mathrm{y}$ ampliación de la laminectomía, apreciándose un desgarro lateral dural en la raíz. El segundo caso ocurrió en un paciente con fijación lumbar L2-L5 manifestándose postquirúrgicamente con déficit motor para la elevación del muslo y extensión de la rodilla derecha, acompañado de un cuadro de disestesias. La Rx y la TAC mostraban posible compromiso medial a nivel del tornillo L3 derecho (figura 1). Se reintervino al paciente retirando dicho tornillo. En ambos casos persistió el déficit motor con dificultad para la deambulación de los pacientes.

B. Persistencia de estenosis

Ocurrió en una paciente con fijación lumbar L4-S1, donde se agravó la sintomatología previa con dolor en miembro inferior derecho sin déficit neurológico. En la TAC se comprobó la adecuada colocación de la fijación y la persistencia de estenosis foraminal a nivel L4-L5 derecho. Por ello se reintervino ampliando la foraminotomía a nivel L4-L5 derecho. La paciente se recuperó completamente de su sintomatología.

C. Desconexión del sistema de fijación

Un paciente con fijación lumbar L4-L5 presentó, al mes de la intervención, un cuadro de dolor en región lumbar de instauración brusca sin irradiación y sin déficits neurológicos. La Rx de columna lumbar mostró la desconexión del dispositivo transverso de la fijación con deslizamiento hacia masa muscular lumbar (figura 2). Se reintervino colocando un nuevo mecanismo transverso.

\section{Complicaciones tardías}

\section{A. Estenosis de los niveles adyacentes a la fijación}

Fueron reintervenidos 8 pacientes por estenosis del nivel inmediatamente superior a la fijación, 6 con fijaciones de 1 nivel (más frecuente en L4-L5 con 4 casos), 1 caso con fijaciones de 2 niveles y otro con instrumentaciones de 3 niveles (tabla 2). Fueron 5 mujeres y 3 hombres con una media de edad de 57,5 años (rango de 50 a 65). En la paciente con fijación L3-L4, además de la alteración del espacio superior, se produjo una estenosis del nivel inferior. La media de tiempo hasta la aparición de la clínica que obligó a la reintervención fue de 3,6 años (rango 1 a 6,6 años). La mayoría de los pacientes presentaron clínica de lumbalgias irradiadas a un miembro o a ambos, la claudicación sólo apareció en dos pacientes no observándose antes de 4 años del inicio de la clínica. Los estudios de imagen mostraron estenosis del nivel superior a la fijación con degeneración discal, pero sin observarse inestabilidad de este segmento (figura $3 \mathrm{izq}$ y dcha). Todas las reintervenciones consistieron en una laminectomía descompresiva del nivel superior de la

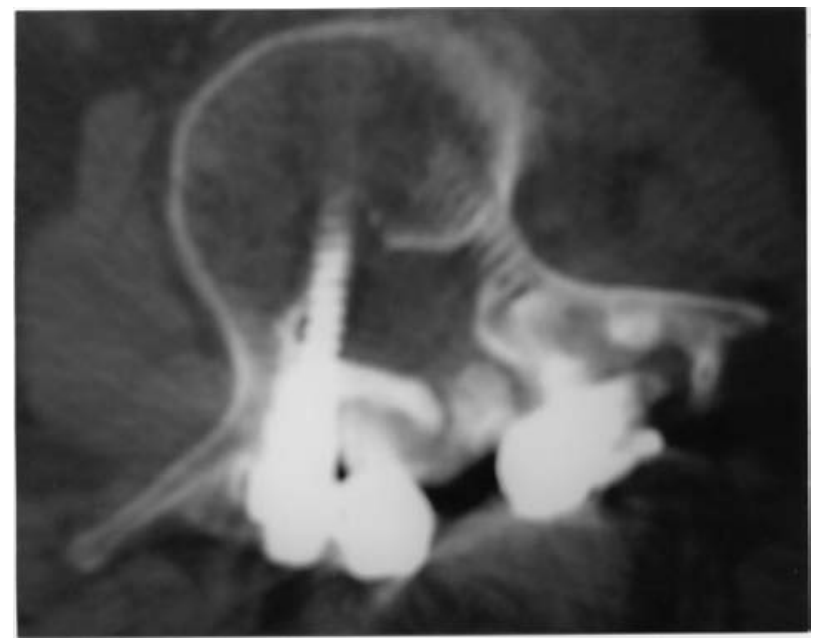

Figura 1. TAC lumbar que muestra malposición de tornillo transpedicular L3 derecho.

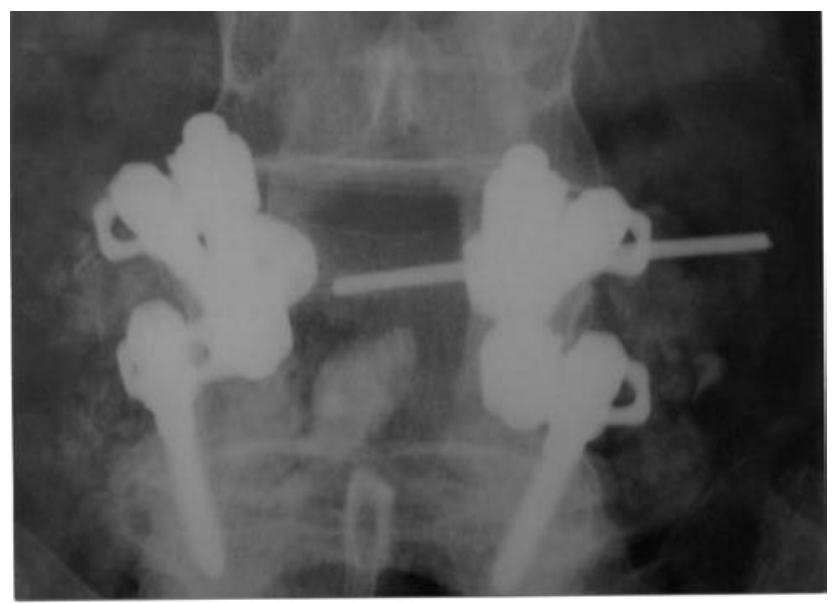

Figura 2. Rx anteroposterior mostrando fallo del sistema transverso.

fijación con fusión posterolateral con hueso autólogo no instrumentada. Se retiraron dos fijaciones en el mismo acto quirúrgico. El cuadro clínico mejoró en todos los pacientes, persistiendo lumbalgias mecánicas crónicas en un paciente.

B. Fibrosis perirradicular

Una paciente con fijación lumbar L5-S1 comenzó con lumbociática izquierda de 5 o 6 meses de evolución con sensación de calambre y déficit motor distal leve. Las pruebas radiológicas demostraron una compresión de las raíces L4 y L5 por material probablemente fibroso. Se intervino quirúrgicamente revisándose la fijación y descomprimiendo las raíces de material fibroso y óseo. La paciente mejoró del cuadro doloroso, persistiendo el déficit motor leve.

C. Rotura de tornillos transpediculares

Una paciente con fijación lumbar L4-L5 presentó 


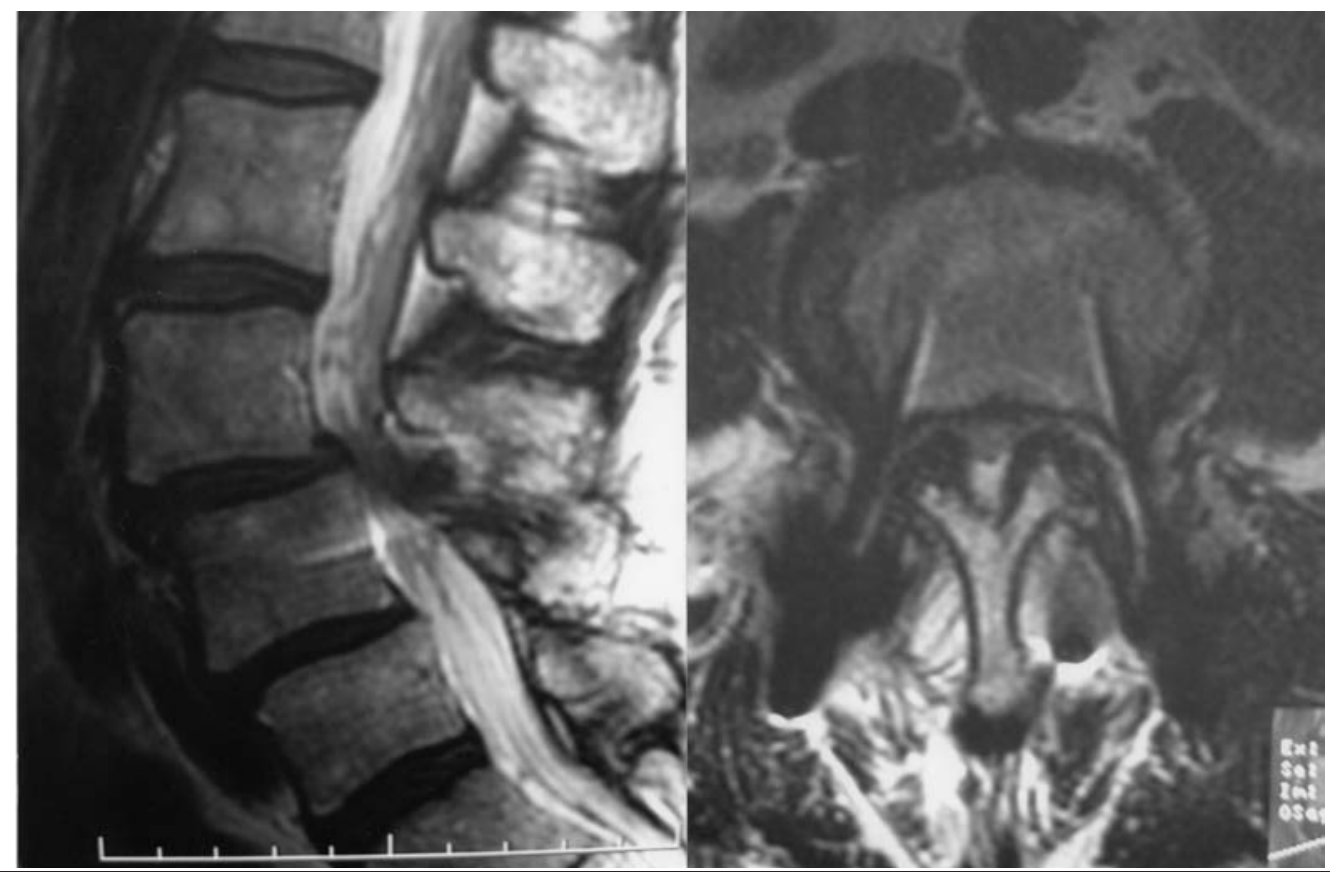

Figura 3. Izquierda: $R M$ sagital potenciada en $T 2$ mostrando estenosis en el nivel superior a la fijación. Derecha: RM axial potenciada en T2 mostrando estenosis superior a la fijación.

Tabla 2

Estenosis del nivel superior a la fijación

\begin{tabular}{|c|c|c|c|c|}
\hline Nivel & años & sexo & Síntomas & tiempo de inicio \\
\hline L3-L4 & 59 & q & Lumbalgia irradiada a cara anterior muslo I. & 2,5 años \\
\hline L5-S1 & 50 & $\hat{0}$ & Lumbociática irradiada por cara exterior & 2,5 años \\
\hline L3-S1 & 57 & $\hat{0}$ & Claudicación. Lumbalgias. & 6 años \\
\hline L4-L5 & 58 & q & Lumbalgias con irradiación a MID. & 6,6 años \\
\hline L3-L5 & 52 & 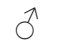 & Lumbalgias irradiadas a MII. & 2 años \\
\hline L4-L5 & 64 & q & Lumbalgias irradiadas a MMII.Claudicación & 4 años \\
\hline L4-L5 & 65 & q & Lumbalgias no irradiadas. & 4,2 años \\
\hline L4-L5 & 55 & $q$ & Lumbociática irradiada a MMII. & 1 año \\
\hline
\end{tabular}

un cuadro de síndrome lumbar con tensión radicular bilateral a $70-80^{\circ}$ de inicio insidioso. La TAC mostró fractura de ambos tornillos L5 (figura 4), por lo que se reintervino ampliando la fijación a S1. La paciente mejoró levemente de su cuadro doloroso.

D. Dolor neuropático

Dos pacientes comenzaron con dolor de características neuropáticas, uno portador de una fijación L4-S1, de inicio al año tras la cirugía, y otro de inicio a los 3 años tras fijación L4-L5. Las pruebas radiológicas mostraron una fusión adecuada sin ningún tipo de patología. En ambos casos se retiró la instrumentación lumbar persistiendo el dolor. Posteriormente se colocó a ambos pacientes un neuroestimulador dorsal a nivel de D11 con mejoría evidente del cuadro doloroso.

\section{Discusión}

Las indicaciones de la colocación de un sistema de fijación transpedicular, en pacientes operados por estenosis de 


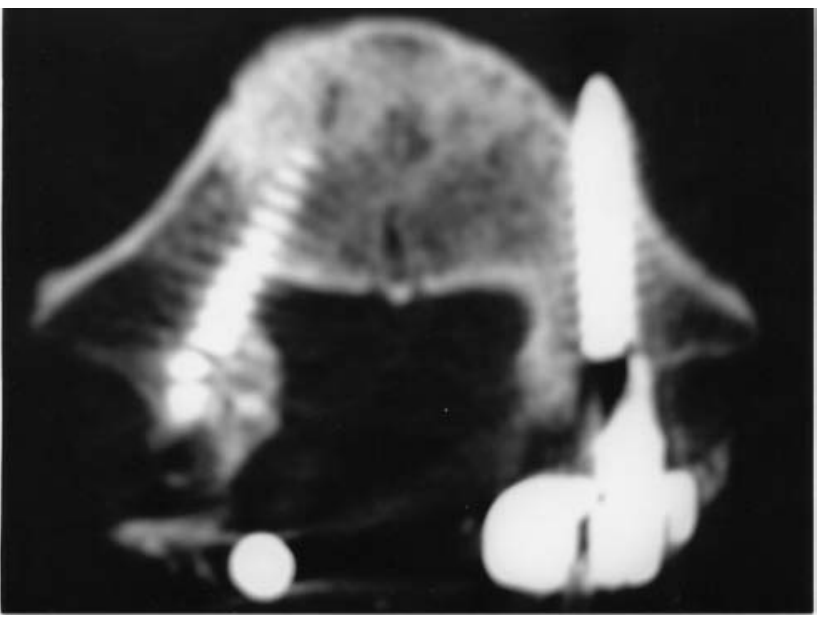

Figura 4. TAC lumbar mostrando fractura de ambos tornillos transpediculares colocados en L5.

canal, en el mismo acto quirúrgico que la descompresión se debe a dos motivos: el primero es tratar la espondilolistesis degenerativa que en frecuentes ocasiones acompaña al cuadro (un 50\% en nuestra serie); el segundo motivo es paliar las amplias descompresiones quirúrgicas de los segmentos estenóticos que crearían una inestabilidad de la zona $^{6,19,34}$.

El tema de la artrodesis lumbar con o sin instrumentación en la estenosis de canal con o sin espondilolistesis degenerativa es controvertido. Se han realizado numerosos estudios en los que se muestran mejores tasas de fusión en pacientes sometidos a una descompresión con fusión instrumentada, sin embargo, con resultados divergentes en cuanto a la mejoría clínica. Bridwell y cols. ${ }^{5}$ realizaron un estudio con 44 pacientes comparando las distintas opciones quirúrgicas en pacientes con estenosis de canal y espondilolistesis degenerativa. Los pacientes instrumentados obtuvieron mejores tasas de fusión (87\%) y mejores resultados clínicos $(83 \%)$ que los pacientes no instrumentados (30\% ambos). Un estudio de Zdeblick $^{43}$ sobre 124 pacientes mostró mejores tasas de fusión en pacientes con instrumentación rígida (95\%), así como en los resultados clínicos (95\%) en comparación con los pacientes no instrumentados (65\% y $71 \%$ respectivamente). Lorenz y cols. ${ }^{29}$, sobre 68 pacientes, observaron una mayor tasa de pseudoartrosis en el grupo no instrumentado (59\%) que en el grupo instrumentado, donde no hubo ningún caso con pseudoartrosis, además, se obtuvo mejor respuesta clínica en este último grupo. Herkowitz y $\mathrm{Kurz}^{21}$ realizaron otro estudio comparativo entre fusión instrumentada y no instrumentada en 50 pacientes, observando tasas de fusión y resultados clínicos. Los resultados fueron superiores en el grupo de pacientes instrumentados, pero los autores reflejan como interesante que un $36 \%$ de los pacientes en los que falló la fusión tuvieron un buen resultado clínico.
Esto hizo pensar a los autores en la hipótesis de la presencia de uniones fibrosas que estabilizan lo suficiente para evitar la inestabilidad y aliviar los síntomas. En el estudio de Fischgrund y cols. ${ }^{15}$ sobre 76 pacientes obtuvieron unas tasas de fusión muy superiores en los pacientes en los que la fijación fue instrumentada (82\%) en comparación con los no instrumentados (45\%), sin embargo, en cuanto a la mejoría clínica los pacientes en los que no se colocó un sistema de fijación fue superior (85\%) en comparación con los pacientes con una instrumentación lumbar $(78 \%)$. Este hecho también hizo hipotetizar sobre la existencia de puentes fibrosos radiotransparentes que estabilizan la zona consiguiendo buenos resultados clínicos. Un metanálisis de la literatura realizado por Mardjetko y cols. ${ }^{31}$ sobre la espondilolistesis degenerativa en 889 pacientes en los que se les practicó descompresión sin fijación, descompresión con fusión posterolateral sin instrumentación y descompresión con fusión instrumentada. Los resultados mostraron mayores tasas de fusión en pacientes instrumentados (93\%) que en los pacientes sin instrumentación, pero los resultados clínicos fueron superiores en pacientes con fusión no instrumentada (90\%) que en los instrumentados (86\%). Thomsen y cols. ${ }^{40}$ en un estudio comparativo en 130 pacientes entre instrumentación y no instrumentación concluyeron que no había diferencias significativas en términos de fusión y mejora clínica. Resultados similares obtuvieron en sus estudios Jäger y cols. ${ }^{24}$, France y cols. ${ }^{17}$, McGuire y Amundson $^{33}$ y Kimura y cols. ${ }^{25}$, concluyendo que no está justificado el uso general de la instrumentación lumbar en pacientes con estenosis de canal y espondilolistesis degenerativa.

En general, la indicación de una instrumentación lumbar basada en la creación de un segmento inestable por amplias descompresiones es difícil de determinar. El estudio de Abumi $^{1}$ y cols. basado en el estudio biomecánico de facetectomías concluye que se crea inestabilidad del nivel con resecciones completas de una faceta articular o con resecciones superiores al 50\% de ambas facetas articulares. Sin embargo, en personas de edad avanzada, debido a la gran degeneración en la columna lumbar y a la formación de osteofitos que aumentan la estabilidad de la zona, es posible acometer amplias descompresiones sin crear inestabilidad de la zona, aunque en casos con espondilolistesis degenerativa, o bien con escoliosis, sería aconsejable la $\operatorname{artrodesis}^{30,39}$. En personas más jóvenes con columnas menos degeneradas, donde la clínica de estenosis de canal que predomina es la estenosis de recesos laterales, cuando se realiza amplias facetectomías es necesaria la colocación de un sistema de fijación lumbar para estabilizar la zona $^{16,21,22}$.

Es conocido que la instrumentación transpedicular puede ser un riesgo para la degeneración de los segmentos adyacentes $^{13,38,42}$. La mayor frecuencia de reintervenciones 
en nuestra serie fue la estenosis de los segmentos superiores con 8 pacientes, siendo el $10,8 \%$ de todos los pacientes y el $50 \%$ de todas las reintervenciones. En otras series esta frecuencia varia del 2 al 17\%, $\%^{4,1326,27,36}$, incluyendo, además de la estenosis, la inestabilidad de dichos segmentos. El tiempo medio de desarrollo de esta patología en nuestro estudio es de 3,6 años (rango de 1 a 6,6 años), mientras que en el estudio de Booth y cols. ${ }^{4}$ el tiempo medio es 7 años (rango de 4 a 12 años). En el estudio de Kumar y cols. ${ }^{26}$ el inicio medio es de 5,2 años, mientras que en el estudio de Wiltse y $\operatorname{cols}^{42}$ el inicio de los síntomas se produjo a los 7 años de media. El grado de lordosis durante la cirugía es muy importante para evitar la degeneración de los espacios adyacentes, en casos con hipolordosis se produce mayor sobrecarga del nivel superior, mientras que la hiperlordosis condiciona una mayor sobrecarga del nivel inferior ${ }^{2,41}$. Cierto grado de fibrosis es esperable después de una intervención quirúrgica, sin embargo, no se suele observar como causa típica de reintervención. Se ha descrito en el $10 \%{ }^{20}$, mientras que en nuestra serie solo se reintervino 1 caso $(1,35 \%)$ considerando que la fibrosis podría haber sido la causa de la clínica.

La fractura de tornillos se produce generalmente a nivel sacro en tornillos de poco diámetro $(5 \mathrm{~mm})$ en la zona del cuello del tornillo, que es la zona más crítica ${ }^{8,10}$. La frecuencia varía dependiendo de las series entre el 1,3 y el $27 \% \%^{4,11,12,25,32,35-37,40}$. En nuestra serie se produjo en 1 paciente $(1,35 \%)$ en ambos tornillos pediculares en la vértebra L5.

Otras complicaciones estarían en relación con problemas de la técnica quirúrgica, viéndose malposición de los tornillos transpediculares en dos de nuestros pacientes $(2,7 \%)$, produciéndose en ambos lesión de las raíces de la que no se recuperaron clínicamente. En otras series la frecuencia de malposición varia del 1 al 10\% ${ }^{11,14,15,20,28,37,40}$, con mayor aumento de las lesiones neurológicas al añadirse las producidas en la maniobra de distracción. Estas varían del 3 al $13 \% \%^{25,35,37}$.

En un paciente $(1,35 \%)$ persistió la estenosis tras la cirugía debido a una inadecuada descompresión, mientras que en otras series es del $2 \%{ }^{20}$.

El fallo del sistema de fijación por aflojamiento del mecanismo transverso se produjo de forma súbita en un paciente $(1,35 \%)$. En otras series revisadas se observó entre el 2 y el $13 \%{ }^{25,37}$.

En estas complicaciones precoces de la fijación transpedicular, sobre todo las lesiones neurológicas por una posible malposición, conllevan la necesaria realización de un estudio de imagen, empezando por una Rx simple, la cual nos aporta poca información. Es necesaria la realización de una TAC de la zona de la instrumentación o una resonancia magnética (RM), sobre todo en secuencias T1, que es donde se observan menos artefactos producidos por el titanio de la fijación".

En nuestra serie no se reintervino a ningún paciente de pseudoartrosis ni de infecciones profundas, que son frecuentes en otras series. La frecuencia de reintervenciones por pseudoartrosis varía del 1 al 20\% $\%^{15,20,37}$, mientras que la frecuencia de infecciones profundas está entre el $1 \%$ y el $10,7 \%$, $411,18,25,35,37,40$.

Además de las complicaciones producidas por la colocación de la instrumentación lumbar se produce otro tipo de complicaciones relacionadas con la elevada edad de los pacientes, donde el mayor tiempo quirúrgico y la pérdida de un mayor volumen sanguíneo hacen que estos pacientes sean más susceptibles a desarrollar tanto complicaciones mayores (infección profunda, neumonía, fallo renal... etc.) como menores (infección urinaria, anemia, confusión, íleo... etc.). Esta mayor incidencia de complicaciones hace que tengamos que poner mayor atención en el control de la pérdida de volumen sanguíneo y en el tiempo quirúrgico ${ }^{7}$.

\section{Conclusiones}

Las complicaciones relacionadas con la técnica quirúrgica son bajas. La mitad de las reintervenciones en pacientes con estenosis del canal lumbar está producida por la estenosis del segmento superior a la fijación. Consideramos que la instrumentación lumbar estaría indicada en pacientes jóvenes con estenosis lumbar en los que se les ha practicado amplias descompresiones con facetectomías bilaterales, así como en pacientes de edad avanzada donde se asocia espondilolistesis degenerativa o escoliosis lumbar.

\section{Bibliografía}

1. Abumi, K., Panjabi, M.M., Kramer, K.M.: Biomechanical evaluation of lumbar spinal stability after graded facetectomies. Spine. 1990; 15: 1142-1147.

2. Akamaru, T., Kawahara, N., Tim Yoon, S.: Adjacent segment motion after a simulated lumbar fusion in different sagittal alignments: a biomechanical analysis. Spine. 2003; 28: $1560-1566$.

3. Arnoldi, C.C., Brodsky, A.E., Cauchoix, J., et al.: Lumbar spinal stenosis and nerve root entrapment syndromes. Definition and classification. Clin Orthop Relat Res. 1976; 115: 4-5.

4. Booth, K.C., Bridwell, K.H., Eisenberg, B.A.: Minimum 5-year results of degenerative spondylolisthesis treated with decompression and instrumented posterior fusion. Spine. 1999; 24: 1721-1727.

5. Bridwell, K.H., Sedgewick, T.A., O'Brien, M.F.: The role of fusion and instrumentation in the treatment of degenerative spondylolisthesis with spinal stenosis. J Spinal Disord. 1993; 6: 461-472. 
6. Brunon, J., Chazal, J., Chirossel, J.P., et al.: When is spinal fusion warranted in degenerative lumbar spinal stenosis? Rev Rhum Engl Ed. 1996; 63: 44-50.

7. Carreon, L.Y., Puno, R.M., Dimar, J.R. $2^{\text {nd }}$. Perioperative complications of posterior lumbar decompression and arthrodesis in older adults. J Bone Joint Surg Am. 2003; 85: 2089-2092.

8. Carson, W.L., Duffield, R.C., Arendt, M., Ridgely, B.J., Gaines, R.W. Jr.: Internal forces and moments in transpedicular spine instrumentation. The effect of pedicle screw angle and transfixation--the 4R-4bar linkage concept. Spine. 1990; 15 : 893-901.

9. Colak, A., Kutlay, M., Demircan, N.: MR imaging for early complications of transpedicular screw fixation. Eur Spine J. 1999; 8: 151-155.

10. Cunningham, B.W., Sefter, J.C., Shono, Y.: Static and cyclical biomechanical analysis of pedicle screw spinal constructs. Spine. 1993; 18: 1677-1688.

11. Davne, S.H., Myers, DL.: Complications of lumbar spinal fusion with transpedicular instrumentation. Spine. 1992; 17: 184-189.

12. Esses, S.I., Sachs, B.L., Dreyzin, V.: Complications associated with the technique of pedicle screw fixation. A selected survey of ABS members. Spine. 1993;18: 22312238 .

13. Etebar, S., Cahill, D.W.: Risk factors for adjacentsegment failure following lumbar fixation with rigid instrumentation for degenerative instability. J Neurosurg. 1999; 90: 163-169.

14. Farber, G.L., Place, H.M., Mazur, R.A.: Accuracy of pedicle screw placement in lumbar fusions by plain radiographs and computed tomography. Spine. 1995 ; 20: 1494-1499.

15. Fischgrund, J.S., Mackay, M., Herkowitz, H.N.: 1997 Volvo Award winner in clinical studies. Degenerative lumbar spondylolisthesis with spinal stenosis: a prospective, randomized study comparing decompressive laminectomy and arthrodesis with and without spinal instrumentation. Spine. 1997; 22: 2807-2821

16. Fox, M.W., Onofrio, B.M., Onofrio, BM.: Clinical outcomes and radiological instability following decompressive lumbar laminectomy for degenerative spinal stenosis: a comparison of patients undergoing concomitant arthrodesis versus decompression alone. J Neurosurg. 1996; 85: 793802 .

17. France, J.C., Yaszemski, M.J., Lauerman, W.C., et al.: A randomized prospective study of posterolateral lumbar fusion. Outcomes with and without pedicle screw instrumentation. Spine. 1999; 24: 553-560.

18. Ghogawala, Z., Benzel, E.C., Amin-Hanjani, S., et al.: Prospective outcomes evaluation after decompression with or without instrumented fusion for lumbar stenosis and degenerative Grade I spondylolisthesis. J Neurosurg Spine. 2004; 1 : $267-272$.
19. Grob, D., Humke, T., Dvorak, J.: Degenerative lumbar spinal stenosis. Decompression with and without arthrodesis. J Bone Joint Surg Am. 1995; 77: 1036-1041.

20. Hadjipavlou, A., Enker, P., Dupuis, P.: The causes of failure of lumbar transpedicular spinal instrumentation and fusion: a prospective study. Int Orthop. 1996; 20: 35-42.

21. Herkowitz, H.N., Kurz, L.T.: Degenerative lumbar spondylolisthesis with spinal stenosis. A prospective study comparing decompression with decompression and intertransverse process arthrodesis. J Bone Joint Surg Am. 1991; 73: 802-808.

22. Herkowitz, H.N.: Lumbar spinal stenosis: indications for arthrodesis and spinal instrumentation. Instr Course Lect. 1994; 43: 425-433.

23. Herkowitz, H.N.: Degenerative lumbar spondylolisthesis. Spine. 1995; 20: 1084-1090.

24. Jäger, M., Seller, K., Raab, P.: Clinical outcome in monosegmental fusion of degenerative lumbar instabilities: instrumented versus non-instrumented. Med Sci Monit. 2003; 9: 324-327.

25. Kimura, I., Shingu, H., Murata, M.: Lumbar posterolateral fusion alone or with transpedicular instrumentation in L4--L5 degenerative spondylolisthesis. J Spinal Disord. 2001; 14: 301-310.

26. Kumar, M.N., Baklanov, A., Chopin, D.: Correlation between sagittal plane changes and adjacent segment degeneration following lumbar spine fusion. Eur Spine J. 2001; 10: 314-319.

27. Kuroki, H., Tajima, N., Kubo, S.: Clinical results of posterolateral fusion for degenerative lumbar spinal diseases: a follow-up study of more than 10 years. J Orthop Sci. 2002; 7: 317-324.

28. Laine, T., Makitalo, K., Schlenzka, D.: Accuracy of pedicle screw insertion: a prospective CT study in 30 low back patients. Eur Spine J. 1997; 6: 402-405.

29. Lorenz, M., Zindrick, M., Schwaegler, P., et al.: A comparison of single-level fusions with and without hardware. Spine. 1991; 16: 455-458.

30. Marchesi, D.G., Aebi, M.: Pedicle fixation devices in the treatment of adult lumbar scoliosis. Spine. 1992; 17: 304309 .

31. Mardjetko, S.M., Connolly, P.J., Shott, S.: Degenerative lumbar spondylolisthesis. A meta-analysis of literature 1970-1993. Spine. 1994; 19:2256-2265.

32. McAfee, P.C., Weiland, D.J., Carlow, J.J.: Survivorship analysis of pedicle spinal instrumentation. Spine. 1991; 16: $422-427$.

33. McGuire, R.A., Amundson, G.M.: The use of primary internal fixation in spondylolisthesis. Spine. 1993; 18: 16621672.

34. Mullin, B.B., Rea, G.L., Irsik, R.: The effect of postlaminectomy spinal instability on the outcome of lumbar spinal stenosis patients. J Spinal Disord. 1996; 9: 107-116. 
35. Narayan, P., Haid, R.W., Subach, B.R.: Effect of spinal disease on successful arthrodesis in lumbar pedicle screw fixation. J Neurosurg. 2002; 97: 277-280.

36. Pfeiffer, M., Hildebrand, R., Grande, M.: Evaluation of indication-based use of transpedicular instrumentations with different rigidity for lumbar spinal fusion: a prospective pilot study with 3 years of follow-up. Eur Spine J. 2003; 12: 369377.

37. Pihlajamaki, H., Myllynen, P., Bostman, O.: Complications of transpedicular lumbosacral fixation for non-traumatic disorders. J Bone Joint Surg Br. 1997; 79: 183-189.

38. Rahm, M.D., Hall, B.B.: Adjacent-segment degeneration after lumbar fusion with instrumentation: a retrospective study. J Spinal Disord. 1996; 9: 392-400.

39. Simmons, E.D. Jr, Simmons, E.H.: Spinal stenosis with scoliosis. Spine. 1992; 17: 117-120.

40. Thomsen, K., Christensen, F.B., Eiskjaer, S.P.: 1997 Volvo Award winner in clinical studies. The effect of pedicle screw instrumentation on functional outcome and fusion rates in posterolateral lumbar spinal fusion: a prospective, randomized clinical study. Spine. 1997 ;22: 2813-2822.

\section{Comentario al trabajo: Causas de reintervención qui- rúrgica en pacientes con fijación lumbar intervenidos por estenosis de canal de Gómez de la Riva y cols.}

Los autores describen las causas de reintervención en una serie de 74 pacientes con estenosis de canal lumbar (ECL) que fueron tratados mediante laminectomía y fijación instrumentada. El $50 \%$ de estos pacientes presentaba una espondilolistesis degenerativa asociada y en el $50 \%$ restante, al parecer, la decisión de fijación se realizó para evitar una inestabilidad posterior secundaria a una amplia laminectomía.

En cuanto al número de reintervenciones (21\%), aunque pueda parecer alto, es similar al reportado en otras series sin instrumentación', si bien es de destacar que en esta serie sólo el $4 \%$ de los pacientes tuvieron que ser reintervenidos para realizar una fusión por inestabilidad. En la serie presentada llama la atención que $6(37,5 \%)$ de los 16 pacientes en los que el segmento $S_{1}$ fue incluido en la fusión tuvieron que ser reintervenidos.

Como mencionan los autores, existe una gran controversia sobre la utilidad de realizar fusión o no en pacientes con ECL y aún más sobre la necesidad de que esta fusión sea instrumentada. En un meta-análisis reciente realizado por Gibson y Waddell ${ }^{2}$ para la Cochrane la conclusión parece clara. La instrumentación mejora de forma significativa el porcentaje de fusión pero no el pronóstico final del paciente y además, existen indicios de que la instrumentación puede
41. Umehara, S., Zindrick, M.R., Patwardhan, A.G.: The biomechanical effect of postoperative hypolordosis in instrumented lumbar fusion on instrumented and adjacent spinal segments. Spine. 2000; 25: 1617-1624.

42. Wiltse, L.L., Radecki, S.E., Biel, H.M., et al.: Comparative study of the incidence and severity of degenerative change in the transition zones after instrumented versus noninstrumented fusions of the lumbar spine. J Spinal Disord. 1999; 12: 27-33.

43. Zdeblick, T.A.: A prospective, randomized study of lumbar fusion. Spine. 1993; 18: 983-991

Gómez de la Riva, A.; Isla, A.; Pérez-López, C.; Ortega, R,; Fernández-Miranda, J.C.; Heredero, J.: Causas de reintervención quirúrgica en pacientes con fijación lumbar intervenidos por estenosis de canal. Neeurocirugía 2006; 17: 232-239

Correspondencia postal: A. Gómez de la Riva. Servicio de Neurocirugía. Hospital Universitario La Paz. Paseo de la Castellana 261, 28046, Madrid.

aumentar el número de complicaciones e incrementar la pérdida hemática quirúrgica, el tiempo quirúrgico, los costes y el número de infecciones. En este sentido, es de destacar la ausencia de complicaciones infecciosas en la serie presentada, que como mencionan los autores, oscila entre $1-10 \%$ en la literatura.

Me parece un artículo muy interesante que nos debe hacer reflexionar sobre la necesidad de realizar estudios randomizados prospectivos que nos ayuden a mejorar la selección de los pacientes que pueden beneficiarse de la instrumentación lumbar.

\section{Bibliografía}

1. Jonson, B., Annertz, M., Sjoberg, C. Stromqvist B.: A prospective and consecutive study of surgically treated lumbar spinal stenosis: Part II: Five-year follow-up by an independent observer. Spine 1997; 22: 2938-2944.

2. Gibson, J.N. Waddell, G.: Surgery for degenerative lumbar spondylosis. Cochrane Database Syst Rev. 2005; 18 : CD001352. 